\title{
Derivative Weighted Active Insulin Control Modelling and Clinical Trials for ICU Patients
}

Carmen V. Doran ${ }^{1}$, Nicolas H. Hudson ${ }^{1}$, Katherine T. Moorhead ${ }^{1}$, $\underline{\text {. Geoffrey Chase }}^{2}$, and Geoffrey M. Shaw ${ }^{3}$

University of Canterbury

Dept of Mechanical Engineering

Private Bag 4800

Christchurch

New Zealand

Email: geoff.chase@canterbury.ac.nz

\footnotetext{
${ }^{1}$ Postgraduate Research Assistant, Dept of Mechanical Engineering, University of Canterbury

${ }^{2}$ Sr. Lecturer, Dept of Mechanical Engineering, University of Canterbury, Christchurch, New Zealand

${ }^{3}$ Consultant, Dept of Intensive Care Medicine, Christchurch Hospital, Christchurch, New Zealand
} 


\section{Abstract}

Close control of blood glucose levels significantly reduces vascular complications in Type 1 and Type 2 diabetic individuals. Heavy derivative controllers using the data density available from emerging biosensors are developed to provide tight, optimal control of elevated blood glucose levels, while robustly handling variation in patient response. A two-compartment glucose regulatory system model is developed for intravenous infusion from physiologically verified subcutaneous infusion models enabling a proof-of-concept clinical trial at the Christchurch Hospital Department of Intensive Care Medicine. This clinical trial is the first of its kind to test a high sample rate feedback control algorithm for tight glucose regulation. The clinical trial results show tight control with reductions of $79-89 \%$ in blood glucose excursions for an oral glucose tolerance test. Experimental performance is very similar to modelled behaviour. Results include a clear need for an additional accumulator dynamic for insulin behaviour in transport to the blood and strong correlation of $10 \%$ or less between modelled insulin infused and the amounts used in clinical trials. Finally, the heavy derivative PD control approach is seen to be able to bring blood glucose levels below the (elevated) basal level, showing the potential for truly tight control.

\section{Introduction}

Diabetes is a disorder of the metabolism whereby either insufficient insulin is produced by the beta cells in the pancreas, or the body is unable to effectively utilise that insulin. As a result, glucose cannot be transported to the cells, leading to dangerously high blood glucose levels. Untreated over time, high blood glucose levels 
can lead to costly complications, including kidney failure, blindness, nerve damage, heart attack and ineffectuality of the immune system. Over 120 million people are affected by diabetes worldwide, and this number is expected to rise to 300 million by the year 2025, with annual costs growing exponentially with the number of cases (Thomson et al., 2001).

Figure 1 schematically shows how blood glucose levels react to a glucose input. Blood glucose rises over the basal level of approximately $4.5 \mathrm{mmol} / \mathrm{L}$ for a normal individual and usually takes 2-3 hours to return to basal. A Type 1 diabetic individual typically has no beta cells and cannot produce any insulin. Uncontrolled, their blood glucose level remains high indefinitely, so an exogenous insulin input is imperative. A Type 2 diabetic individual either produces insufficient insulin or insulin which is ineffective in taking glucose out of the blood, hence their blood glucose returns to a typically elevated basal level more slowly, requiring exogenous insulin in severe cases. Both Type 1 and Type 2 diabetic individuals may also have elevated blood glucose basal levels (Votey and Peters, 2001 and 2002).

Current treatment of Type 1 and severe Type 2 diabetes involves regular point monitoring of the plasma glucose level and injecting insulin into the subcutaneous tissue as required. Using a glucose monitoring system and an insulin pump or syringe injections, all current treatments are performed manually by the patients themselves or, in a hospital setting, under professional care. Therefore, diabetic individuals are encouraged to regularly monitor food intake and daily activity to help maintain blood glucose levels at an acceptable value. 
Existing insulin pumps and emerging non-invasive and semi-invasive glucose monitoring systems could be interconnected and a closed loop system realized. Ultimately, the control unit should be able to automate $90-95 \%$ of a diabetic's dayto-day insulin care, freeing the patient from the anxieties and stress of constantly monitoring their blood glucose regulatory system behaviour, allowing them to lead more "normal" lives. In addition, the acquisition and storage of data on a regular basis will provide far better input for clinicians.

Substantial research in understanding, modelling and managing diabetes has led to no shortage of theoretical automated solutions (e.g. Doyle et al., 1995; Ollerton et al., 1989; Kienitz et al., 1993; Fisher et al., 1991; Trajonoski et al., 1995; Cobelli et al., 1999; Furler et al., 1985; Bergman et al., 1985; Kraegen et al., 1984; Cobelli et al., 1982). However, due to either the complexity of the proposed implementation, current technological limitations, models that are not physiologically verified, lack of required data, or the cost/complexity of realising the results, these solutions do not yet seem feasible. Several researchers have examined the analysis and automation of insulin administration as reviewed by Lehman et al. (1996). Many of the systems presented use the mathematics of control systems as a means of providing clinical advice or testing the effectiveness of a new protocol (e.g. Schrezenmeir et al., 1991; Skyler et al., 1981; Lehman et al., 1993; Boroujerdi et al., 1987; Fletcher et al., 1991). Optimal control using grid search theory, robust $\mathrm{H}$-infinity control, and variable structure controllers have also been studied, using various models (e.g. Ollerton et al., 1989; Kienitz et al., 1993; Fisher et al., 1991; Berger et al., 1991; Parker et al., 1996; Naylor et al., 1995). In each case, the focus has been on controlling absolute blood glucose excursion rather than the shape of the glucose curve. The models used 
typically require either several patient specific parameters that are not generically available and/or knowledge of glucose or exercise inputs that would not be known à priori. Finally, none of the studies examines the impact of the far more frequent measurements enabled by recent advances in sensing technology or the potential for improved results enabled by these technologies.

Prior work in tightly controlling elevated blood glucose levels using heavy derivative control employed a physiologically verified three compartment model based on the work of Bergman et al. (1985). The three compartments represent insulin production/infusion dynamics, insulin transport from the subcutaneous infusion site, and glucose input and utilisation dynamics. Performance was shown to improve as the sensor sampling period and sensor lag decrease, and the control solution is seen to reduce the magnitude and duration of blood glucose excursion in response to glucose challenge more effectively than a normal human body when blood glucose levels are measured with a sample period of 1 minute (Lam et al., 2002; Chase et al., 2002a). Finally, optimal infusion solutions were also developed for cases in which the glucose infusion profile is well known (Chase et al., 2002b).

The model presented here develops heavy derivative blood glucose control to a proofof-concept clinical trial with Intensive Care Unit (ICU) patients. An additional benefit to using ICU patients is that they often present with elevated blood glucose levels and tight control of these levels can reduce mortality up to $45 \%$ (Van den Berghe et al, 2001). Related tests have been conducted into modelling glucose control on endogenous glucose production (Simeoni et al., 1997). The clinical results are 
compared to predicted values for verification of the simple models and control methods employed.

\section{Clinical Trials}

The proof-of-concept clinical trials developed are designed to effectively simulate a true feedback control system with a 15 minute sampling period. Prior research into heavy derivative control has shown that a sampling period of 15-20 minutes provides very good performance and represents a technologically realistic level of system performance (Chase et al., 2002a; Lam et al., 2002). Hence, the 15 minute sampling period for these trials was selected to emulate a realistic system, and at 4 measurements per hour the trials procedure will not stress the medical staff involved. Note that the trials are conducted specifically to test the effectiveness of the heavy derivative control methods and to verify the simulations and design that led to them. The clinical trials are conducted on ICU patients, as they represent a highly controlled test group who often experience elevated blood glucose levels.

Qualifying patients for the trial had to be stable, have elevated blood glucose levels over $8 \mathrm{mmol} / \mathrm{L}$, have an arterial line and a nasogastric feed, and be expected to remain in the ICU for at least three days. In addition, patients with morbid obesity (BMI > $35 \mathrm{~kg} / \mathrm{m}^{2}$ ) or neuromuscular blockade were not considered. Ethics approval for these trials was granted by the Canterbury Ethics Committee.

The clinical trials presented are a two-day procedure for each participant. The first day of the trial is uncontrolled and provides data on the glucose regulatory system 
response to an Oral Glucose Tolerance Test (OGTT). The second day of trials involves active insulin control of the glucose regulatory system response to an OGTT using the heavy derivative control algorithm.

\section{Clinical Trial Day One:}

The trial begins at 0700 hours at which time the patient is fasted for four hours. Blood glucose readings are taken every hour to determine the basal blood glucose level. At 1100 hours, blood is taken for C-peptide and blood insulin tests to screen for insulin contamination and to determine the basal insulin level, respectively. The patient is then given a 75g OGTT glucose dose via their nasogastric tube. Plasma glucose is measured at 15-minute intervals until 1500 hours. Paired samples are taken, with one analysed using a bedside Glucocard ${ }^{T M}$ Test Strip II glucose testing kit and the other sent to the laboratory for comparison. The error in these results is approximately $7 \%$ for the Glucocard ${ }^{T M}$ Test Strip II tests, and 3\% for the laboratory tests at typical elevated blood glucose levels (Arkray Inc., 2001; Phillips et al., 1994; Peters et al., 1996). Note that some insulin may be infused on day one for safety or other medical reasons.

\section{Clinical Trial Day Two:}

The procedure is repeated as per day one, however short acting soluble insulin with $0.2 \mathrm{U} / \mathrm{ml}$ in $0.9 \%$ saline is infused via an intravenous cannula using a Graseby 3500 syringe pump. Plasma glucose is measured at 15 minute intervals as previously and the insulin infusion rate is manually adjusted every 15 minutes according to the heavy derivative control algorithm. This approach is designed to specifically test the control 
algorithm by emulating feedback control, while ensuring safety by keeping a human "in the loop".

The first patients presented here employ controllers created using patient specific parameters determined from their uncontrolled (day one) OGTT test results. Later patients would ideally use an "average" set of parameters and resulting control gains for which the model used best represents the average OGTT response for that type of patient.

\section{Mathematical Modelling of the Glucose Regulatory System}

To implement tight glucose control using an automated insulin infusion system for patients in an ICU requires a simple model of the glucose regulatory system. The model must account for intravenous insulin infusion. It must also capture the fundamental dynamics of the rise and fall of blood glucose that it is desired to control.

\subsection{Model}

The initial physiologically verified model employed originated from the work of Bergman et al. (1985), and was used in the development of heavy derivative control for subcutaneous insulin infusion (Lam et al., 2002). This three-compartment model utilises the concept of a remote compartment for the transport of insulin from the subcutaneous infusion site to its utilisation in the blood to reduce blood glucose levels. Patients in the ICU have direct arterial/venous lines (AV Fistula) that bypass the subcutaneous layer present in the three compartment model. To capture the essential 
dynamics, only two compartments are therefore needed. The first to model the rate of insulin uptake into the blood and the second to model the level of blood glucose including insulin mediated transport of glucose from the blood. Therefore, two equations can be written for plasma glucose and insulin concentration, respectively.

$$
\begin{aligned}
& \dot{G}=-p_{1} G-p_{4} I\left(G+G_{B}\right)+P(t) \\
& \dot{I}=-n\left(I+I_{B}\right)+u(t) / V_{I}
\end{aligned}
$$

where :

$$
\begin{array}{ll}
G & =\text { concentration of the plasma glucose above the basal level }\left(\mathrm{mmol} \mathrm{L}^{-1}\right) \\
G_{B} & =\text { basal level for plasma glucose concentration }\left(\mathrm{mmol} \mathrm{L} \mathrm{L}^{-1}\right) \\
I & =\text { concentration of the plasma insulin above basal level }\left(m U L^{-1}\right) \\
I_{B} & =\text { basal level for plasma insulin concentration }\left(m U L^{-1}\right) \\
P(t) & =\text { exogenous glucose infusion rate }\left(m M o l L^{-1} \mathrm{~min}^{-1}\right) \\
u(t) & =\text { exogenous insulin infusion rate }\left(m U \mathrm{~min}^{-1}\right) \\
V_{I} & =\text { insulin distribution volume }(L) \\
p_{1} & =\text { fractional clearance of glucose at basal insulin }\left(\mathrm{min}^{-1}\right) \\
p_{4} & =\text { insulin sensitivity }\left(\mathrm{L} m U^{-1} \mathrm{~min}^{-1}\right) \\
n & =\text { first order decay rate constant for insulin in plasma }\left(\mathrm{min}^{-1}\right)
\end{array}
$$

The parameters, $p_{1}$ and $p_{4}$ may be varied to represent different conditions of the glucose regulatory system. The parameter $p_{4}$ is the insulin sensitivity ratio between $p_{3}$ and $p_{2}$ in Bergman et al (1985), and couples the blood insulin level to the rate of glucose utilization by the body, representing the simplest possible dynamic between these two compartments. Additional model dynamics linking the two compartments in 
Equations (1) and (2) may be required, however any missing dynamics are expected to have little effect on the ability to derive an effective controller.

More specifically, the rise of the glucose concentration is most significant in the first 30-60 minutes where the heavy derivative controller has greatest effect. Hence, this rise is not significantly affected by $p_{4}$ if the insulin infused by the controller in this time frame is not large, such as during the first day of the clinical trial method. It is this rise that the heavy derivative control is focused on limiting, while the subsequent drop with its negative slope is little affected (Lam et al, 2002). Note that the value of $p_{4}$ will impact the glucose level if significant exogenous insulin is present, such as on the controlled second day of the clinical trials.

\subsection{Heavy Derivative Control Input}

The controller determines the amount of exogenous insulin infusion rate at every 15 minute sample time, so $u(t)$ is a discrete function. The specific controller employed is a heavy derivative proportional-derivative (PD) controller defined:

$$
\begin{aligned}
& \mathrm{u}(\mathrm{t})=\max \left(0, U_{0} *\left(1+K_{p} *\left(G+G_{\text {prime }}\right)+K_{d} * d G / d t\right)\right) \\
& G_{\text {prime }}=G_{B}-G_{t}
\end{aligned}
$$

Here $U_{0}$ is the basal infusion level, typically $1 \mathrm{U} / \mathrm{hr}, \mathrm{G}_{\text {prime }}$ is an offset term so that a patient with a high basal glucose level, $G_{B}$, can have their glucose level controlled to a lower target blood glucose level, $G_{t}$, by increasing $G_{\text {prime }}$. For heavy derivative control the proportional gain $K_{p}$ is $20-50$ times smaller than the derivative gain $K_{d}$ 
(Lam et al., 2002). The max function with argument " 0 " ensures that negative insulin demands are treated as a zero input. This zero input demand typically occurs during the fall of blood glucose where additional insulin is not desired to avoid hypoglycemic episodes (Lam et al, 2002).

The fundamental reason for using heavy derivative control, where the derivative gains are significantly higher then the proportional gains, is to control the shape of the blood glucose response rather than its specific magnitude. This approach is an inherently safer method of control than attempting to strictly control just the magnitude of the glucose response. Temporarily high blood glucose levels are not dangerous in the majority of people. However, a hypoglycaemic drop in blood glucose below 3.5-4 mmol/L is dangerous even for a short period of time. Heavy derivative control safeguards against this eventuality by infusing no insulin when the blood glucose level is dropping steeply, a precursor to a hypoglycaemic episode. This behaviour arises from the much larger derivative feedback gain used and the negative blood glucose slope combining to demand a negative infusion $u(t)$. Similarly, it also enables much faster response to rapidly increasing blood glucose levels seen in the first 30-60 minutes of the OGTT. This discussion is summarised schematically in Figure 3.

\subsection{Glucose Infusion Model}

The model output is highly dependant upon the exogenous glucose infusion, $P(t)$, particularly on day one when there is little or no exogenous insulin infusion. However, glucose uptake into the blood from the stomach is highly patient specific. 
To capture the exponential rise and slightly slower exponential fall in blood glucose for an OGTT a lognormal function is used for $P(t)$ as it captures the essential physiological behaviour.

$$
P(t)=\mathrm{x}_{1} \exp \left(-\mathrm{x}_{2}\left(\log \left(\mathrm{x}_{3} \mathrm{t}\right)-\mathrm{x}_{4}\right)^{2}\right)+\mathrm{x}_{5}
$$

where constants $\mathrm{x}_{1}$ to $\mathrm{x}_{5}$ are determined so that the area under the curve is equivalent to $34 \mathrm{mmol} / \mathrm{L}$, a value obtained by converting the $75 \mathrm{~g}$ OGTT glucose dose on the assumption that the patient has the glucose evenly distributed in a $12 \mathrm{~L}$ fluid volume. In addition, the peak of the $P(t)$ curve is set to occur at approximately $80 \%$ of the patients blood glucose peak from day one of the clinical trial to account for patient specific rates of uptake. This assumption is made so that the simulated and actual exogenous glucose uptake rates for the uncontrolled OGTT will be similar, simplifying the process of fitting patient specific parameters from the day one trial data.

Lognormal or similarly shaped exponential functions for glucose uptake have been used by Trajanoski and Wach (1998), Erzen et al (2000), and Kienitz and Yoneyama (1993), and are shown schematically in basic texts (Guyton, 1996). Other authors use exponential functions starting at an elevated glucose level to model OGTT uptake (Fisher and Teo, 1989; Fisher, 1991). Deutsch et al. (1990) define glucose uptake profiles that are effectively trapezoidal approximations of a lognormal shape. Finally, Callegari et al (2002), Furler et al (1985), Ollerton (1989) and others avoid this modelling issue by modelling glucose uptake as the result of a constant, steady state infusion where it is a therefore constant. However, this constant input does not 
capture the transient dynamics of the glucose-regulatory system that it is desired to control. It should also be noted that Bergman et al (1985), among others, present OGTT glucose response data that is lognormal in its fundamental shape for normal individuals and has a similar exponential rise for Type 1 diabetic individuals.

It is therefore important to note that the assumed form of the glucose input, $P(t)$, in the absence of other choices, also means that the modelled glucose response for the uncontrolled day one trial will be essentially lognormal in shape since the transient response is zero with $G(0)=G_{b}$ used. To truly simulate a glucose input from a meal or OGTT challenge, a form for the glucose input must be assumed and the lognormal shape captures the essential exponential data found in clinical data. As a result, the model fitted to the day one trial data will have a generically lognormal form. This choice therefore leads to the loss of some smaller dynamic effects that may be present, but still captures the essential blood glucose rise and fall on which the controller being tested acts (Lam et al, 2002). Note that significant exogenous insulin would change this lognormal shape, as seen on day two of the clinical trials.

\subsection{Determining Patient Specific Parameters}

The system model given by Equations (1) and (2) is patient specific and must be adapted to each patient before a controller can be developed for that patient. Hence, controller design is accomplished in three fundamental steps:

1. Data from an uncontrolled OGTT is gathered from the patient (day one). 
2. The patient specific parameters, $p_{1}$ and $p_{4}$, in the uncontrolled model are obtained via an unconstrained optimisation problem so that the model output approximates the fundamental dynamics of the patient's uncontrolled OGTT curve.

3. Using the patient specific parameters, heavy derivative control gains are developed using a second unconstrained optimisation problem.

Fitting the patient specific parameters from day one of the trial is done in three basic steps. First, the blood glucose peak time is obtained from the data and a glucose input model, $P(t)$, is created for that patient. This patient specific input will dominate the glucose response of the uncontrolled, day one model with little or no exogenous insulin input.

Second, the noisy patient data are fitted to a second lognormal function with emphasis on capturing the blood glucose rise in the first 30-60 minutes and the overall fundamental rise and fall dynamics of the day one blood glucose data. This second step is not the standard approach used in the minimal model literature when determining insulin sensitivity or other parameters from an intra-venous glucose tolerance test (IVGTT). As noted with the creation of a glucose input model, $P(t)$, some non-fundamental dynamics are ignored in the process. However, this approach eliminates the difficulty in finding converged solutions to the optimisation/fitting problem, which can be very difficult with limited (16 points), noisy data as in this case. It also provides a function to which the model parameters can be fitted, providing effectively more points in the area of interest, the rise of blood glucose, rather than evenly spread points. It bears repeating that this approach is non-standard 
due to the use of the glucose input function, $P(t)$, and its impact on the uncontrolled model dynamics for day one, as well as the desire to only capture the fundamental shape of the response, particularly over the first 60 minutes.

The third step uses the function fitted to the day one data to find the parameters $p_{1}$ and $p_{4}$ via unconstrained optimisation using a penalized quadratic objective function defined:

$$
R=\left(\bar{G}_{\text {model }}-\bar{G}_{\text {patient }}\right)^{T}\left(\bar{G}_{\text {model }}-\bar{G}_{\text {patient }}\right)+\mathrm{e}^{-\mathrm{p}_{1} C}+\mathrm{e}^{-\mathrm{p}_{4} C}
$$

where the first term is a vector dot product designed to minimise the difference between the model output, $\bar{G}_{\text {model }}$, and curve fitted experimental data, $\bar{G}_{\text {patient }}$, at the same time points. The parameter $C$ is a large positive constant, defined to ensure that $p_{1}$ and $p_{4}$ remain positive. This approach enables more points to be used in total and notably in the regions of interest. Typically, several extra time points around the peak of the glucose response curve are used to ensure the rise and inflection of the glucose curve is adequately captured for controller design.

Again, the limitations of the model and the parameters as fitted in this fashion are such that it is only useful for capturing the fundamental dynamics and for control design focused on the blood glucose rise in response to the OGTT input. It should also be noted that this optimisation problem is non-convex and therefore multiple starting points were tested to ensure that the best (lowest value of $R$ ) potential fit was obtained.

\subsection{Determining Control Gains}


Once the patient specific model has been created, a controller can be developed to regulate the patient's glucose level. The control gains are determined by minimising a penalized, quadratic objective function that minimises the area between the controlled glucose response and the target glucose level, $G_{t}$. As a result it seeks to minimise the overall blood glucose excursion rather than a particular peak value or final value. The penalty terms are similar to those in Equation (6) and constrain the gain values to be positive. This optimisation-based approach is employed for simplicity to efficiently search a large potential solution space.

\section{Clinical Results}

Clinical trials were performed on four ICU patients meeting the study requirements Table 1 gives the patient age, condition, insulin levels, basal and peak glucose levels, and patient specific parameters resulting from day one of the trial. Table 1 shows that the four patients display a diverse range of glucose responses from a relatively flat response to an extremely volatile reaction. Patients 3 and 4 display relatively low and high insulin levels respectively, although the very high levels for patient 4 were obtained with a partially contaminated sample from the insulin infusion line and are assumed to be higher than the actual value. Conversely, patients 1 and 3 are in the normal range of $5-80 \mathrm{pmol} / \mathrm{L}$. None of the patients had an exceptionally high or low body mass index per the study requirements.

The insulin sensitivity values, $p_{4}$, are of the same order or higher than existing data for sub-cutaneous delivery (Bergman et al, 1981, Avogaro et al 1989). However, sub- 
cutaneous infusions can be subject to up to $20 \%$ losses in transportation (Kraegen and Chisholm, 1984) and are also highly variable in effective delivery of all subcutaneous infused insulin to blood plasma (Home et al, 1982). These losses would be accounted for in this type of model by a reduced sensitivity value, $p_{4}$, and for IV infusion such higher values as used in these trials might be expected. These higher values in comparison to IVGTT data might also be a result of the method of fitting and in particular the use of several starting points for this non-convex optimisation problem.

It should be noted that ICU patients, while often sedated and in a highly controlled state, are actually extremely diverse in the causes and dynamics of their hyperglycaemia. ICU patients experience elevated blood glucose levels due to the stress of their condition, as well as due to any explicitly diagnosed Type 1 or Type 2 diabetes. As a result their response to an OGTT glucose challenge can vary extremely, as noted in Table 1, due to equally extreme variations in insulin levels, effective insulin utilization, glucose absorption, and a variety of other factors. In contrast, the dynamics of a "pure" Type 1 diabetic are far easier to model and, in response to glucose challenge, far easier to predict than those of hyperglycaemic ICU patients in general. Hence, the ICU patient tests presented represent a fairly extreme test of the ability of the models and control systems developed.

\subsection{Patient 1}

The 67 year old female subject had been in the ICU for three days suffering from kidney failure. The kidneys can remove up to $30 \%$ of effective insulin, so kidney 
failure is an "insulin sparing" condition and is one reason for the flatter uncontrolled day one response in Table 1 (Charpentier et al., 2000). The patient was on a dialysis machine on day one of the trial, however it was removed for day two. The patient had high fluid levels which would have affected the glucose absorption dynamics of her body. C-peptide tests were unavailable until after the test was completed, however the basal insulin level of $70 \mathrm{pmol} / \mathrm{L}$ was slightly higher than expected, though still in the normal range. The patient was therefore both hyperglycaemic and somewhat hyper-insulinaemic.

Figure 4 shows the measured and model predicted glucose response for day one (uncontrolled) and day two (controlled). The measured data is presented with the $7 \%$ error associated with GlucoCard ${ }^{\mathrm{TM}}$ II measurements. The summary in Table 2 shows that both the magnitude and duration of blood glucose excursion from the basal level are reduced by at least $50 \%$. The target sub-basal glucose level was not reached over the period of the trial, as the derivative control was not effective when the tail of the curve flattens off. The desire to drive the glucose concentration down to a target level below the patient's basal level is an example of the need for gain scheduling or a modified control approach in this flat response regime.

Overall, the automated algorithm provided rapid, effective control of the OGTT input with no additional insulin in comparison to normal protocols. The predicted response was an extremely good match for the measured data. The difference in day one and day two basal levels is primarily due to changes in feeding and insulin administration over the night between the tests, and is repeated, to some extent, with each patient. Finally, the patient's blood glucose concentration began to increase steadily back to 
$10 \mathrm{mmol} / \mathrm{L}$ over the 2-3 hours after the test when hospital staff returned to their regular sliding scale protocol showing the need for, and efficacy of, this type of automated approach.

\subsection{Patient 2}

The 48 year old male tetraplegic with Acute Respiratory Distress Syndrome (ARDS) had been in the ICU for 72 days. The patient exhibited extremely variable response to most medications and this experience was reiterated during the trial. Due to the patient's extremely high glucose levels and staff experience, a basal insulin infusion of $2 \mathrm{U} /$ hour, rather than the normal $1 \mathrm{U} /$ hour, was maintained throughout fasting and the trial on day one, and accounted for in the modelling process.

As shown in Figure 5 the patient's glucose absorption profile was much faster on day two compared to day one. The slow absorption on day one is attributed to delayed gastric emptying. The patient was aspirated and had an empty stomach on day two resulting in more rapid absorption of the glucose dose. The response on day two also shows the effect of an insulin accumulation dynamic, which becomes apparent after 200 minutes, with the reasonably constant glucose level dropping with no increase in insulin infusion.

Laboratory tests gave basal insulin levels of $59 \mathrm{pmol} / \mathrm{L}$ (day one) and $63 \mathrm{pmol} / \mathrm{L}$ (day two), which are relatively normal. This result implies the patient was not effectively utilising the insulin in the body similar to a Type 2 diabetic response, allowing his 
glucose levels to reach a peak of $24.5 \mathrm{mmol} / \mathrm{L}$ during the uncontrolled oral glucose tolerance test on day one.

Local hospital protocol generally sets the insulin infusion rate at a maximum of $6 \mathrm{U} / \mathrm{hr}$. Due to the high glucose levels and its rapid rise following the OGTT dose, the control algorithm commanded up to $37 \mathrm{U} / \mathrm{hr}$ for a given 15 minute period. Figure 6 shows the insulin infusion profile for patient 2 . The insulin infusion profile demanded by the heavy derivative control algorithm in response to the OGTT dose was efficient in returning the patient swiftly to the basal level. The result is a profile which looks much like an insulin injection combined with a steady infusion. Therefore, the automated infusion profile from the heavy derivative control algorithm matches the current treatment approach of injections for meals on top of steady basal infusion (Lam et al., 2002; Chase et al., 2002a; Gonzalez-Michaca et al., 2002; Boland et al., 1999; Pickup et al, 2002).

Note that the zero infusion at 45 minutes in Figure 6 is due to a sensor error that reported $8.4 \mathrm{mmol} / \mathrm{L}$ when a laboratory result reported $11.6 \mathrm{mmol} / \mathrm{L}$. The control algorithm therefore did not infuse any insulin due to the erroneous large negative slope reported. Laboratory data was higher than the GlucoCard data, although still not inline with the data points only 15 minutes either side of the sensor error. Inadvertently, this result shows the robustness of the controller, as the effects of this low data point on the derivative term were compensated for in the next change in insulin infusion rate. 
Figure 7 shows the measured and predicted results from day two of the trial using a glucose input profile that accounts for the faster glucose absorption on day two and in which an accumulation dynamic is approximated. Withholding a portion of the commanded insulin input for 75 minutes approximates the accumulation dynamic well enough to determine if a better match to the trial data can be obtained. The predicted curve and measured data match much better in this case due to the change in glucose absorption rates and the additional dynamic. Overall, this figure shows the need for the accumulator dynamic to account for the delayed action of insulin, as well as the robustness of the heavy derivative control approach to absorption rate and time. The latter result is due to the heavy derivative controller's emphasis on controlling the shape of the glucose response rather than its magnitude, making it robust to the specific time or rate of glucose absorption (Lam et al., 2002).

\subsection{Patient 3}

The 75 year old male patient had been in the ICU for 2 days suffering from an intracerebral bleed resulting from a head injury. Uncontrolled patient data in Figure 8 shows that the patient behaves as a Type 1 diabetic, although records stated that it had not been diagnosed. The extremely high glucose levels and extended, almost permanent period of excursion from the basal glucose level are similar to a Type 1 diabetic response to an OGTT and the laboratory tests for insulin level supported this assumption with a value of $3 \mathrm{pmol} / \mathrm{L}$.

The day two measured data in Figure 8 also shows an accumulation dynamic. To approximate this dynamic the addition of a 75 minute delay on a portion of the insulin 
infusion, as with patient 2, resulted in an output that was more closely aligned with the controlled patient data than the original model prediction. Although this approximation does not exactly model the accumulation and delay in the utilisation of insulin, it further confirms the need for this dynamic in the model. Overall, the peak excursion from the basal level is reduced from $6.7 \mathrm{mmol} / \mathrm{L}$ to $2.7 \mathrm{mmol} / \mathrm{L}$, a reduction of 59.7\%. The duration of blood glucose excursion was cut to two hours from the much greater than four hour excursion in the uncontrolled case.

\subsection{Patient 4}

The fourth patient was a 59 year old female, suffering from sepsis and infection, who had been in the ICU for three days after being transferred from another hospital as the infection progressed. Figure 9 shows an almost flat glucose response curve in the day one trial. This flat curve along with the higher than normal glucose level implies the patient was both hyperglycaemic, due to the elevated blood glucose level, and hyperinsulinaemic. With the lack of a significant increase in glucose levels from basal, and hence the low derivative values impacting the heavy derivative PD controller output, the level of infusion was effectively constant on day two of the trial. The sub-basal target glucose level was chosen to be $1 \mathrm{mmol} / \mathrm{L}$ below the basal level on day two of the trial at $5.4 \mathrm{mmol} / \mathrm{L}$. The control algorithm proved to be very efficient at obtaining this level, even with the very low proportional control component of the controller. Figure 9 shows an initial dip in the measured data and simulation output on both controlled and uncontrolled data that can be attributed to delay in glucose uptake.

\subsection{Summary}


A comparison between the predicted insulin infusion profile and actual insulin profile for day two of the trials were made as a means for determining the effectiveness of the modelling methods. Table 2 shows that the total insulin infused over four hours differed from the predicted insulin infusion total by no more than $12 \%$, with an average error of approximately 3\% over the four trials. This strong correlation between the test and simulation results for such a diverse variety of patients helps to validate the fundamental models and methods employed. Many of the differences, where the model tends to undershoot, can be attributed to either one of two factors. The first is the discrete $0.2 \mathrm{U} / \mathrm{mmol}$ discrete insulin infusion level verses exact analogue values available in the model. The second is that for large doses, such as was seen with patient 2, some insulin may be "lost" along the length of the Graseby infuser tubing.

To obtain a better estimate of the control effectiveness, the excursion is quantified as the sum of the area of the blood glucose curve, using the measured data, above the basal glucose level as illustrated in Figure 10. The ratio between the controlled and uncontrolled excursion areas from the clinical trial data is used to determine the overall effect of the control algorithm. The reduction in the glucose basal level on day two of the trial measures the improvement obtained as the controller aims for the lower target basal glucose level. Table 3 summarises the excursion reduction and basal reduction ratios. For all four patients the excursion from basal blood glucose level has been reduced by $79 \%$ to $89 \%$, and the basal glucose level has been reduced $12 \%$ to $41 \%$. 
Finally, the accumulator dynamic noted in the clinical results has three potential causes. The first is the physiological battle between the body's desire to return to its (elevated) basal level and the controller's attempts to reduce it, as best seen at the end of the response in Figure 4. Second, the demand for insulin in the blood is secondary to those of the brain and liver, leading to the possibility that meeting these latter demands first causes a reduction in useful insulin in the blood and a later over reaction (Cherrington et al., 2002; Toschi et al., 2002). Finally, it is believed that insulo-penic, or very low insulin level, patients can develop lipo-toxicity, suppressing insulin release from any active beta cells. Therefore, when exogenous insulin is infused and blood glucose drops, these beta cells are freed to release endogenous insulin not initially modelled (Del Prato et al, 2002; Xiao et al., 2002; Sobngwi et al., 2002). In this last case, additional tests to determine the ratio of C-peptide levels and insulin levels following the test could be compared to the same ratio taken prior to the test to check for a rise in the endogenous insulin produced. Further tests will help clarify the specific causes of this dynamic, and improve the models and clinical trial methods employed.

\section{Conclusions}

These trials have proven very successful at illustrating the effectiveness and potential of automated insulin administration and the heavy derivative approach to active insulin control. The first four proof-of-concept clinical trials approved have succeeded in the creation and validation of an intravenous insulin infusion model and in demonstrating tight, actively controlled blood glucose level regulation. The heavy derivative control approach employed has been demonstrated to be as effective in 
practice as it was in simulation, with reductions in blood glucose excursion of up to $89 \%$ and basal glucose level reductions of up to $41 \%$.

The four trials have shown a high level of correlation between the simulation model and the patient results verifying the fundamental models and methods. Demonstrations of the control algorithm's robustness to glucose absorption rate and absorption profile have been made. However, the results have also clearly demonstrated the need for additional dynamics in the intravenous glucose regulatory system model to accommodate the effects of insulin accumulation in the system. The need for gain scheduling or a two-stage controller to bring elevated blood glucose levels to a lower basal value as blood glucose derivative values become negligible in the tail of the OGTT response has also been noted.

Finally, model specific results include the development and validation of a simple glucose regulatory system model for the intravenous infusion of insulin. A method of rapidly identifying patient specific parameters from curve fitted experimental patient data using unconstrained optimisation was presented. The clinical trial results show that the modelling methods employed, while not standard, did effectively capture the fundamental dynamics operated on by the simple controller tested, however, as noted, some potentially important dynamics may have been ignored in the process.

Future work will be directed toward improving the models and methods employed with an overall goal of even tighter, more robust control. A major focus will be developing better optimisation and fitting methods to reduce the reliance on fitted data, rather than the sparse data collected. In particular, modified trials that rely more on 
insulin challenges rather than glucose challenges will alleviate the need for modelling $P(t)$ and having to therefore assume some form of the response.

\section{Acknowledgements}

The authors wish to acknowledge Prof. Don Beaven, Dr. Helen Lunt, Professor Russell Scott and Jinny Willis PhD of the Canterbury District Health Board and Otago School of Medicine in Christchurch for their support and input to this work. Additional thanks are due Dr. Anne Peters, Dr. Mayer Davidson, Dr. David Schriger and Dr. Vic Hasselblad for gladly sharing data from their research (Peters et al, 1996).

Funding for this research has been provided by the Intensive Care Medicine Trust at Christchurch Hospital and Diabetes New Zealand.

\section{References}

Arkray Inc. 2001 “Glucocard ${ }^{\mathrm{TM}}$ Test Strip 2 Data Sheet”, Supplied by Arkray, Inc. Japan

Avogaro, Angelo., Bristow, James D., Bier, Dennis M., and Cobelli, Claudio., (1989) "Stable-Label Intravenous Glucose Tolerance Test Minimal Model”, Diabetes, vol 38, pp. $1048-1055$

Berger, M. and Rotbard D. (1991). "A pharmacodynamic approach to optimising insulin therapy," Computer Methods and Programs in Biomedicine, vol 34, pp. 241-253. 
Bergman, Richard N., Phillips, Lawrence S., and Cobelli, Claudio., (1981) "Physiological Evaluation of Factorss Controlling Glucose Tolerance in Man", Journal of Clinical Investigation, vol 68, pp.1456-1467

Bergman, Richard N., Finegood, D. T., and Ader, M., (1985) "Assessment of Insulin Sensitivity in Vivo", Endocrine Reviews, vol 6, no 1, pp 45- 86.

Boland EA, Grey M, Oesterle A, Fredrickson L, Tamborlane WV. "Continuous subcutaneous insulin infusion. A new way to lower risk of severe hypoglycaemia, improve metabolic control and enhance coping in adolescents with type I diabetes," Diabetes Care 1999 Nov;22(11):1779-84.

Boroujerdi, M., Williams, C., Carson, E., Piwernetz, K., Hepp, K., and Sonksen, P. (1987). "A simulation approach for planning insulin regimes," Intl. Symposium on Advanced Models for Therapy of Insulin Dependent Diabetes, pp. 41-46.

Callegari, T, Caumo, A and Cobelli, C (2002). "Generalisation of a Map Estimation in SAAM II: Validation Against ADAPT II in a Glucose Model Case Study," Annals of Biomedical Engineering, Vol. 30, pp. 961-968.

Charpentier G, Riveline JP, Varroud-Vial M. Management of drugs affecting blood glucose in diabetic patients with renal failure. Diabetes Metab 2000 Jul;26 Suppl 4:73-85

Chase, J G, Lam, Z-H, Lee, J-Y and Hwang, K-S (2002a). “Active Insulin Infusion Control of the Blood Glucose Derivative," Proc. of the 7th International Conf on Control, Automation, Robotics and Vision (ICARCV 2002), Singapore, Dec 3-5.

Chase, J G, Wake, G C, Lam, Z-H, Lee, J-Y, Hwang, K-S, and Shaw, G M (2002b). “Steady State Optimal Insulin Infusion for Hyperglycemic ICU Patients," Proc. of 
the 7th International Conf on Control, Automation, Robotics and Vision (ICARCV 2002), Singapore, Dec 3-5.

Cherrington A D., Sindelar D., Edgerton D., Steiner K., MxGuinness O P., "Physiological Consequences of Phasic Insulin Release in the Normal Animal". Diabetes 2002 51: S130-108.

Cobelli, C., Deferspil, G., Pacibi, G., Salvan, A., and Scandellari, S. (1982) “An Integrated Mathematics Model of the Dynamics of Blood Glucose and Its Hormonal Control” Mathematical Biosciences, vol 58 pp 27-60.

Cobelli, C., Nucci, G., and Del Prato, S. (1999). "Physiological simulation model of the glucose-insulin system," IEEE Conf. on Engineering in Medicine and Biology, Oct 16, pp. 999.

Del Prato S, Marchetti P, Bonadonna RC "Phasic insulin release and metabolic regulation in type 2 diabetes," Diabetes 2002 Feb; 51 Suppl1:S109-16.

Deutsch, T, Carson, E R, Harvey, F E, Lehman, E D, Sonksen, P H, Tamas, G, Whitney, G, and Williams, C D (1990). "Computer-assisted Diabetic Management: A Complex Approach," Computer Methods and Programs in Biomedicine, Vol. 32, pp. 195-214.

Doyle, F., Dorski, C., Harting, J., and Peppas, N. (1995). "Control and modeling of drug delivery devices for the treatment of diabetes," Proc. of the American Control Conference, June 21-23, pp. 776-780

Erzen, F C, Birol, G and Cinar, A (2000). "Simulation Studies on the Dynamics of Diabetes Mellitus," IEEE International Symposium on Bio-Informatics and Biomedical Engineering, Nov 8-10, pp. 231-235. 
Fisher, M E., (1991). "A Semiclosed-Loop Algorithm for the Control of Blood Glucose Levels in Diabetics", IEEE Transactions on Biomedical Engineering, vol 38, No. 1 pp 57-61

Fisher, M E and Teo, K L (1989). "Optimal Insulin Infusion Resulting from a Mathematical Model of Blood Glucose Dynamics," IEEE Trans on Biomedical Engineering, Vol. 36(4), pp. 479-486.

Fletcher, L. and Soden, P. (1991). "Diet construction using linear programming," Diabetes, Nutrition and Metabolism, Supplement 1, pp. 169-174.

Furler, S M., Edward, W, Smallwood, R H., and Chisholm, Donald J. (1985). "Blood Glucose Control by Intermittent Loop Closure in the Basal Mode: computer Simulation Studies with a Diabetic Model", Diabetes Care, Vol. 8, No. 6 pp 553 561.

Gonzalez-Michaca L., Ahumada M., Ponce-de-Leon S. "Insulin subcutaneous application vs. continuous infusion for postoperative blood glucose control in patients with non-insulin-dependent diabetes mellitus," Arch Med Res 2002 JanFeb; 33(1): 48-52.

Guyton, A C and Hall, J E (1996). "Textbook of Medical Physiology," Elsevier Science, Ninth Edition, Philadelphia, PA.

Home, P.D., Massi-Benedetti, M., Gill, G.V., Capaldo, B., Shepherd, G.A., and Alberti, K.G., (1982). 'Impaired subcutaneous absorption of insulin in 'brittle' diabetics", Acta Endocrinology, vol 101, pp. 414-420

Kienitz, K. and Yoneyama, T. (1993). "Robust controller for insulin pumps based on H-infinity theory," IEEE Trans. On Biomedical Engineering, vol. 40(11), pp. $1133-1137$. 
Kraegen, E. W. and Chisolm, D. J., (1984), "Insulin responses to carting profiles of subcutaneous insulin infusion: kinematics modelling studies", Diaetologia, vol. 26, pp 208-213.

Lam, Z-H, Lee, J-Y and Hwang, K-S, Chase, J G, and Wake, G C (2002) "Active Insulin Infusion Using Optimal and Derivative-Weighted Control”, J. Medical Engineering and Physics, Vol. 24(10), pp. 663-672.

Lehman, E. and Deutsch, T. (1993). "AIDA: a mk. II automated insulin dosage advisor," Journal of Biomedical Engineering, vol 15, pp. 201-211.

Lehman, E. and Deutsch, T. (1996). "Computer assisted diabetes care: a 6-year retrospective," Computer Methods and Programs in Biomedicine, vol 50, pp. 209230.

Naylor, J., Hodel, A., Morton, B., and Schumacher, D. (1995). "Automatic control issues in the development of an artificial pancreas," Proc. ACC, pp. 771-775.

Ollerton, R. (1989). "Application of optimal control theory to diabetes mellitus," Intl. Journal of Control, vol. 50(6), pp. 2503-2522.

Parker, R., Doyle, F., Harting, J., and Peppas, N. (1996). "Model predictive control for infusion pump insulin delivery," IEEE Conf. Of Engineering in Medicine and Biology, Nov 3, pp. 1822-1823.

Peters, Anne L., Davidson, Mayer B., Schriger, David L., Hasselblad Vic. (1996). “A Clinical Approach for the Diagnosis of Diabetes Mellitus," JAMA, vol 276, No. 15, pp. 1246-1252.

Phillips, D.I.W., Clark, P.M., Hales, C.N., Osmond, C. (1994). "Understanding Oral Glucose Tolerance: Comparison of Glucose or Insulin Measurements During the 
Oral Glucose Tolerance Test with Specific Measurements of Insulin Resistance and Insulin Secretion,” Diabetic Medicine, vol. 11, pp. 286-292.

Pickup J, Keen, H. “Continuous subcutaneous insulin infusion at 25 years: evidence base for the expanding use of insulin pump therapy in type I diabetes," Diabetes Care 2002 Mar; 25(3):593-8.

Schrezenmeir, J. (1991). "Computer aids in dietetics," Diabetes, Nutrition and Metabolism, Supplement 1, pp. 175-191.

Simeoni, M., Caumo, A., Rizza, R., Cobelli, C. (1997). "Modelling glucose control on endogenous glucose production on state: necessity of direct and delayed signalling," Engineering in Medicine and Biology Society, 1997. Proceeding of the 19 International Conference of the IEEE, vol 5, pp 2138-2141.

Sobngwi, E., Mauvais-Jarvis, F., Vexiau, P., Mbanya, JC., Gautier, JF., "Diabetes in Africans. Part 2: Ketosis-prone atypical diabetes mellitus". Diabetes \& Metabolism. 28(1):5-12, 2002 Feb.

Skyler, J., Skyler, D., Seigler, D. and Sullivan, M. (1981). "Algorithms for adjustment of insulin dosage," Diabetes Care, vol 4, pp. 311-318.

Thomson, S., Beaven, D., Jamieson, M., Snively, S., Howl, A., and Christophersen, A. (2001). "Type 2 Diabetes: Managing for Better Health Outcomes," PriceWaterHouse-Coopers Report, Diabetes New Zealand Inc.

Toschi E., Camastra S., Sironi A.M., Masoni A., Gastaldelli A., Mari A., Ferrannini E., Natali A., "Effect of Acute Hyperglycemia on Insulin Secretion in Humans". Diabetes 2002 51: S130-133. 
Trajonoski, Z. and Wach, P. (1995). "Evaluation of subcutaneous route for the closedloop control of insulin delivery: Numerical and experimental studies," IEEE Conf. on Engineering in Medicine and Biology, Sep 20-23, pp. 1357-1358.

Trajonoski, Z. and Wach, P. (1998). "Neural Predictive Controller for Insulin Delivery Using the Sub-Cutaneous Route," IEEE Trans on Biomedical Engineering, Vol. 45(9), pp. 1122-1134

Van den Berghe, G., Wouters, P., Weekers, F., Verwaest, C., Bruyninckx, F., Schietz, M., Vlasselaers, D., Ferdinance, P., Lauwers, P., and Bouillon, R. (2001). "Intensive insulin therapy in critically ill patients," New England J. of Med., vol. 345(19), pp. 1359-1367.

Votey, R. S. and Peters, A. L. (2001). "Diabetes Mellitus, Type 1 - A Review," eMedicine Journal, Vol. 2(4), http://www.emedicine.com/EMERG/topic133.htm.

Votey, R. S. and Peters, A. L. (2002). "Diabetes Mellitus, Type 2 - A Review," eMedicine Journal, Vol. 3(4), http://www.emedicine.com/EMERG/topic134.htm.

Xiao, J., Gregersen, S., Pederson, S. B., Hermansen, K., "Differential impact of acute and chronic lipotoxicity on gene expression in INS-1 cells". Metabolism: Clinical \& Experimental. 51 (2): 155-62, 2002 Feb. 


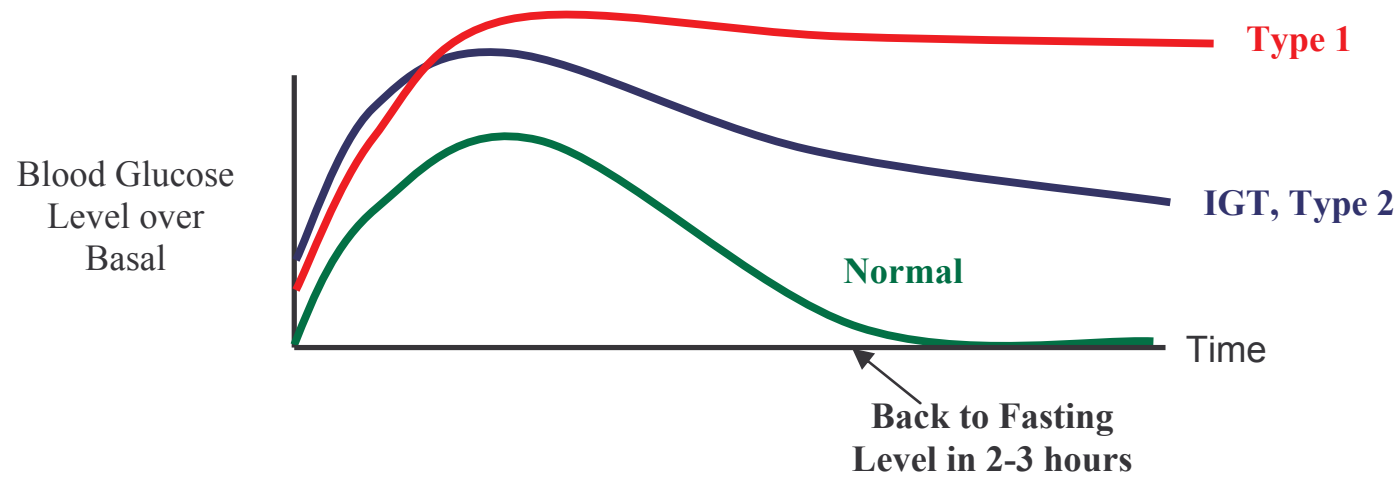

Figure 1: Profile of Blood Glucose Levels immediately after a OGTT 


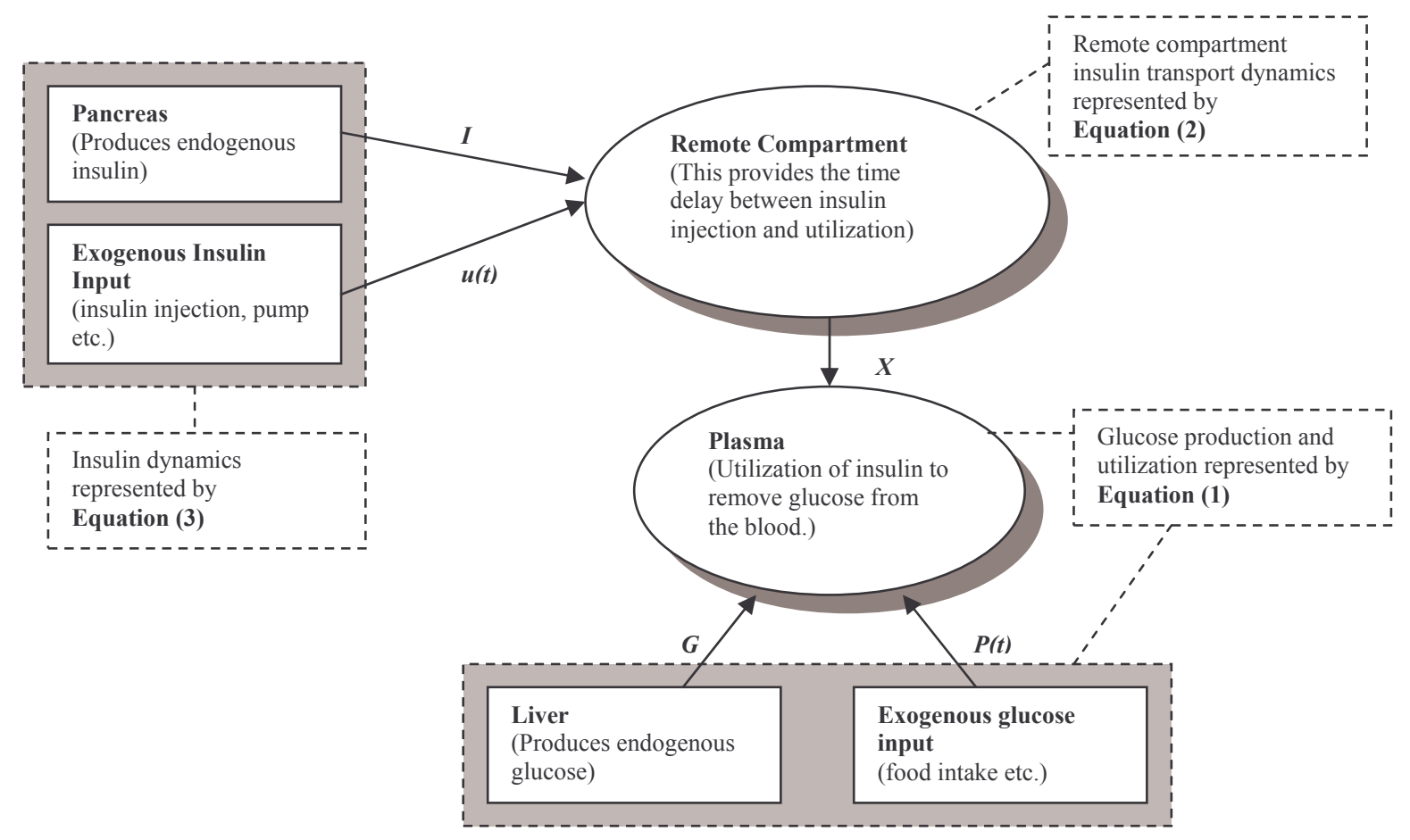

Figure 2: Schematic of the basic dynamics of the human glucose regulatory system. 


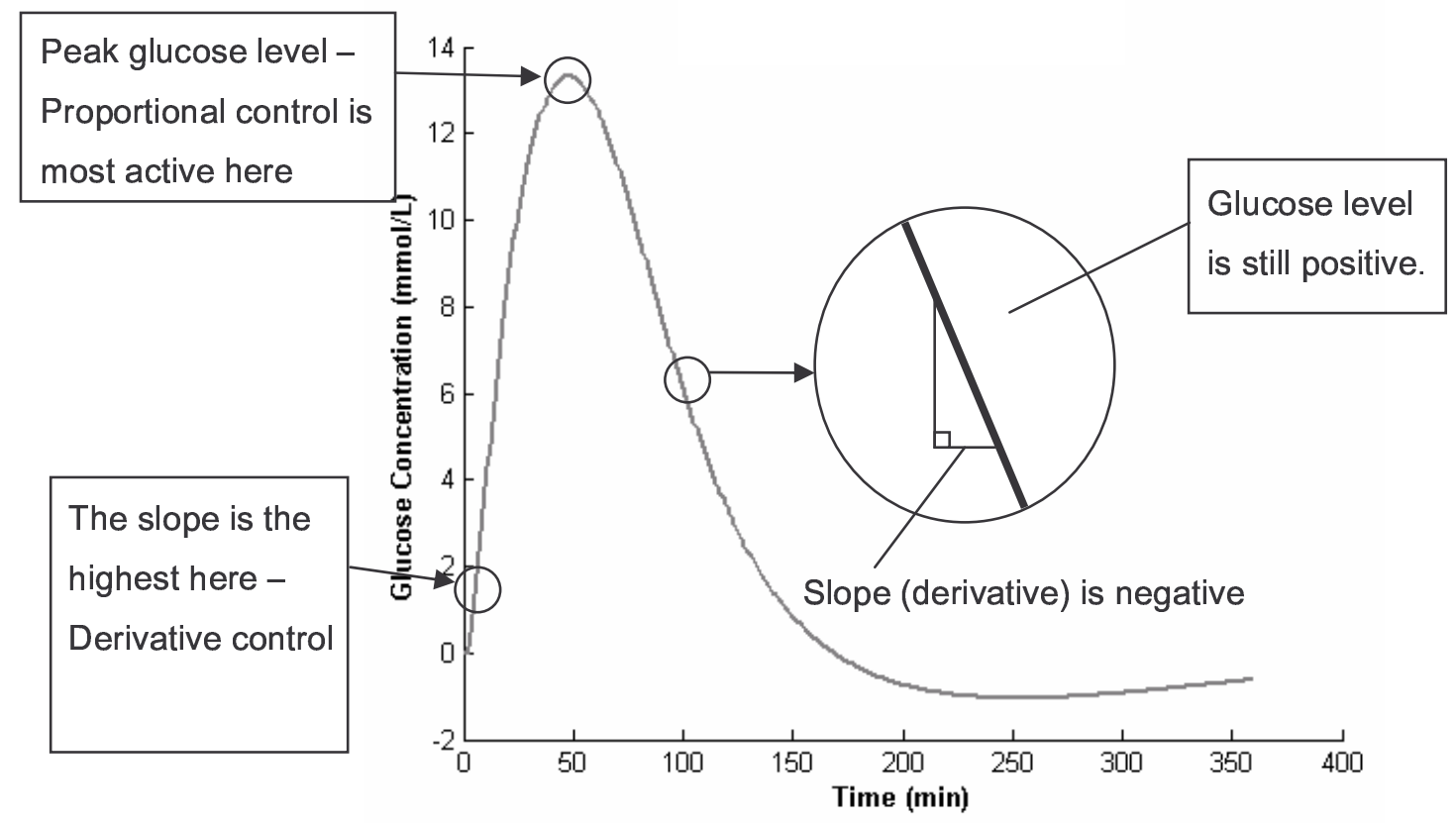

Figure 3: Response Regimes for PD Blood Glucose Control 


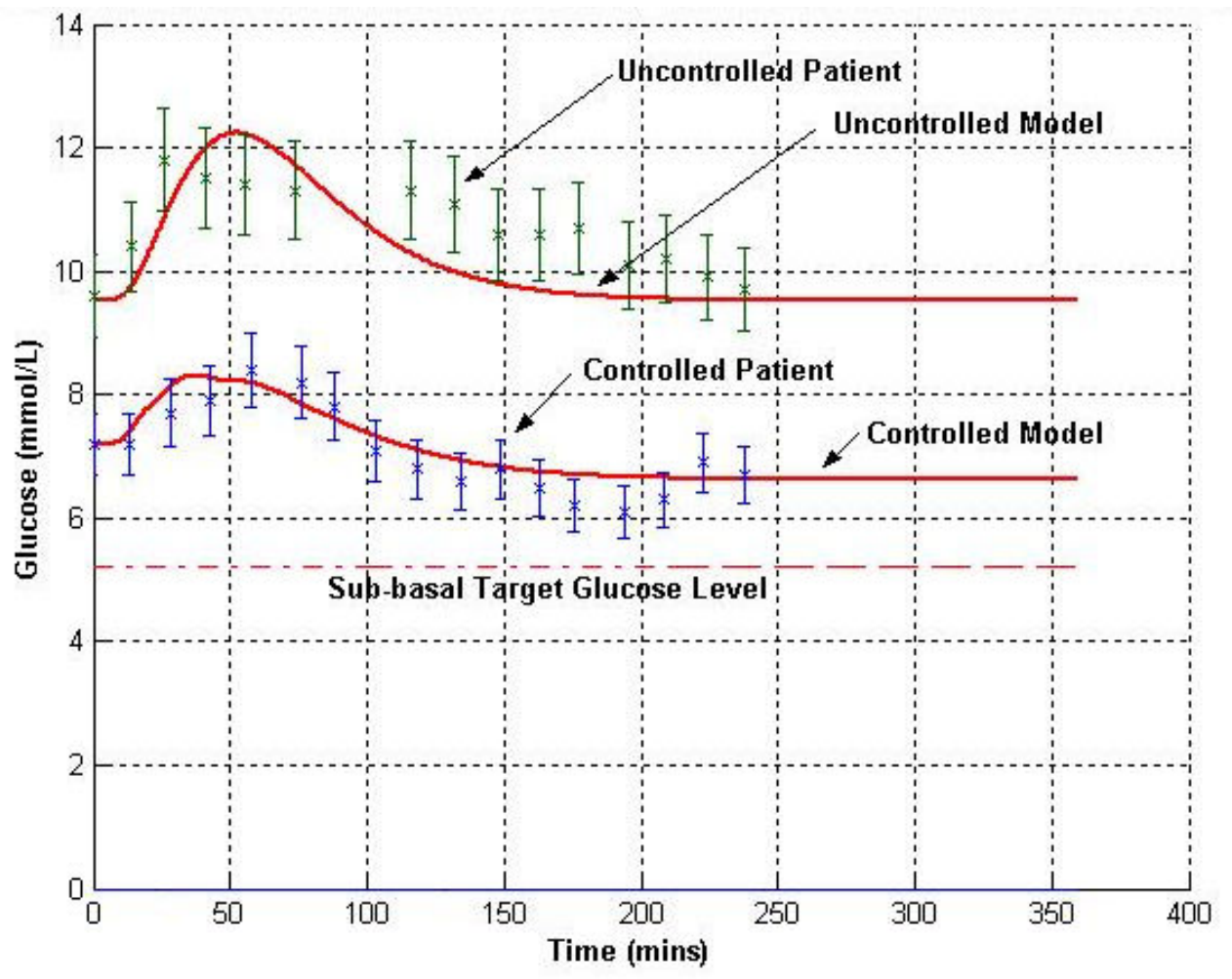

Figure 4: Patient 1 Model vs Measured Glucose - Controlled \& Uncontrolled 75g OGTT 


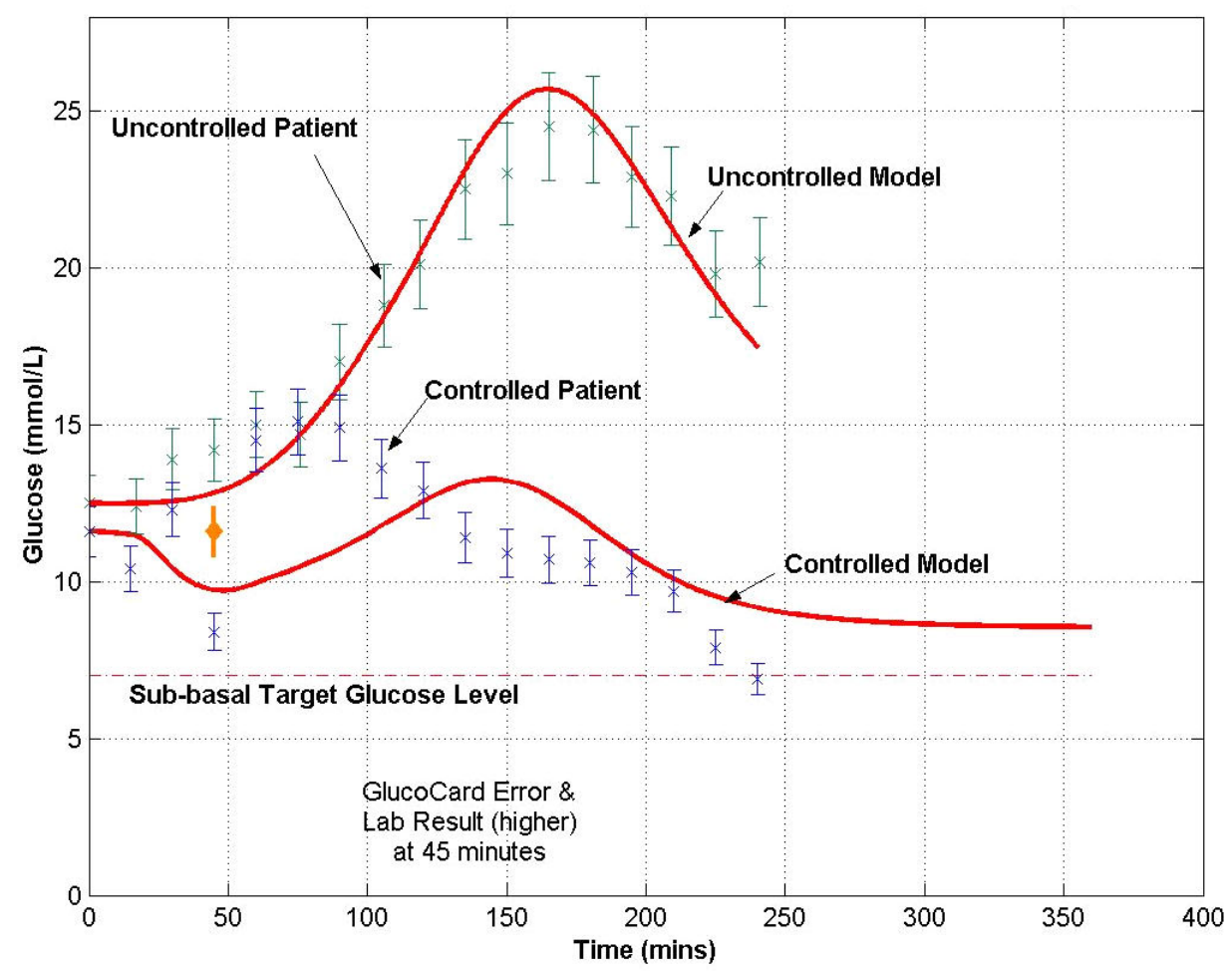

Figure 5: Patient 2 Model vs Measured Glucose - Controlled \& Uncontrolled 75g OGTT 


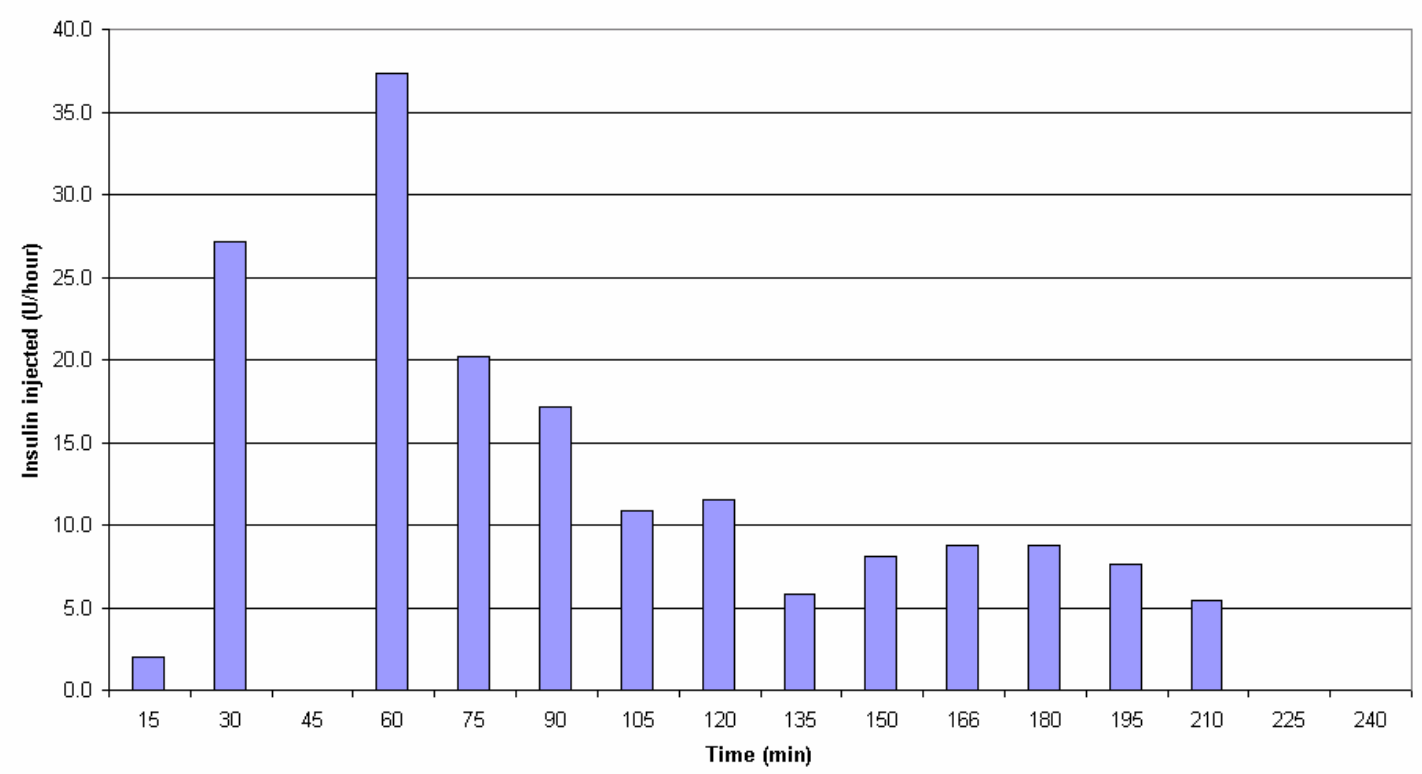

Figure 6: Patient 2 Insulin Infusion Profile 


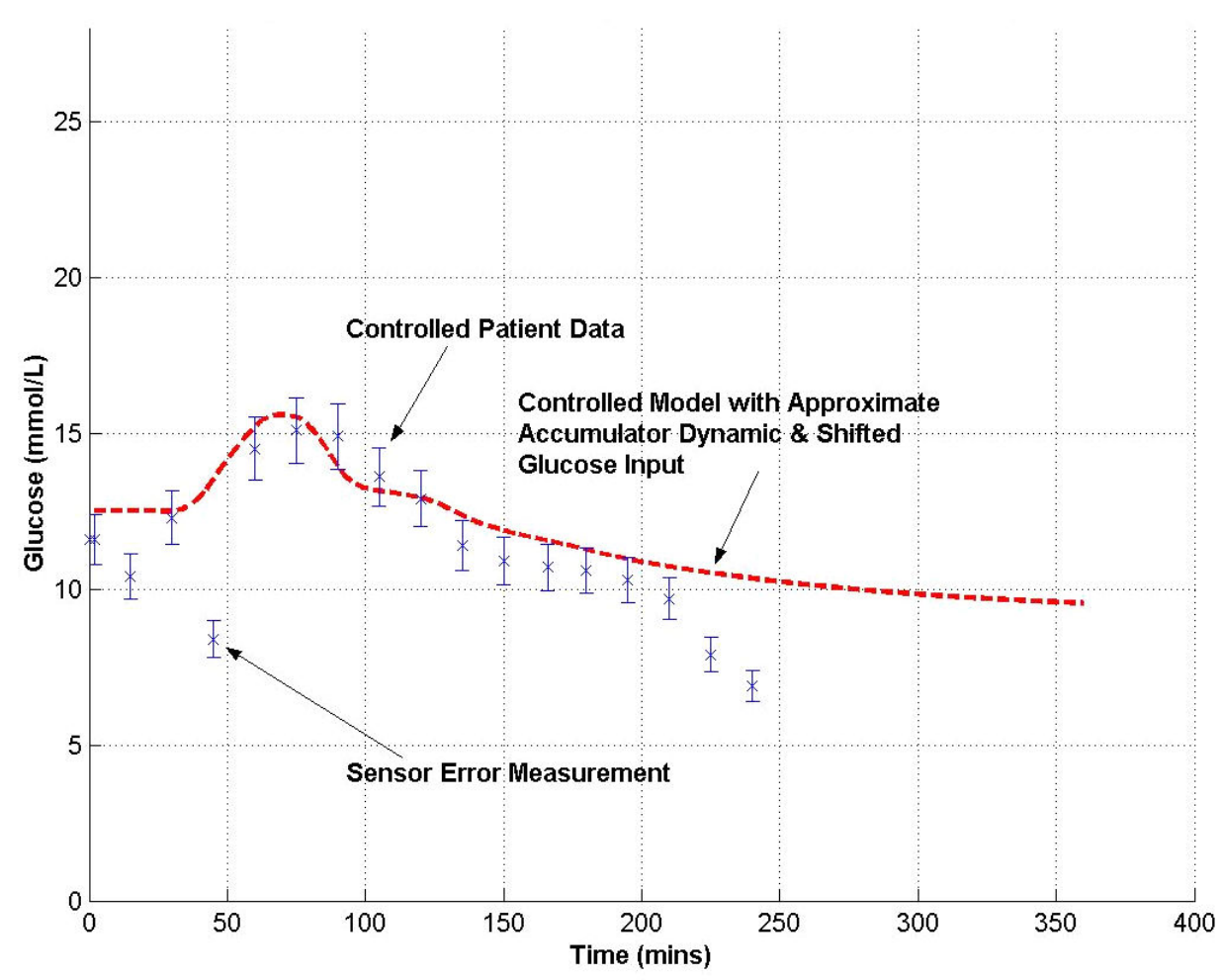

Figure 7: Patient 2 Glucose Response with Shifted Glucose Input 


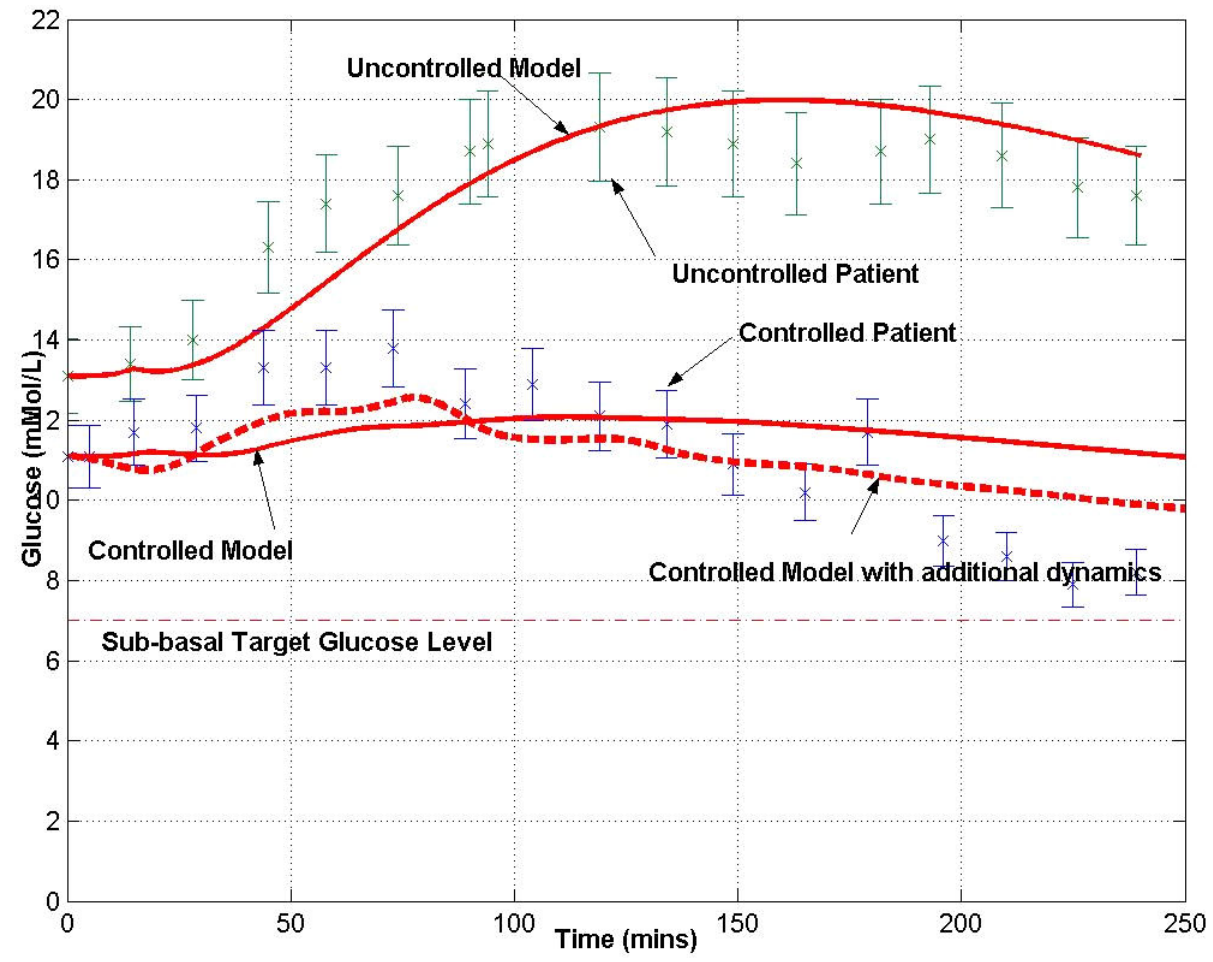

Figure 8: Patient 3 Model vs Measured Glucose - Controlled \& Uncontrolled 75g OGTT 


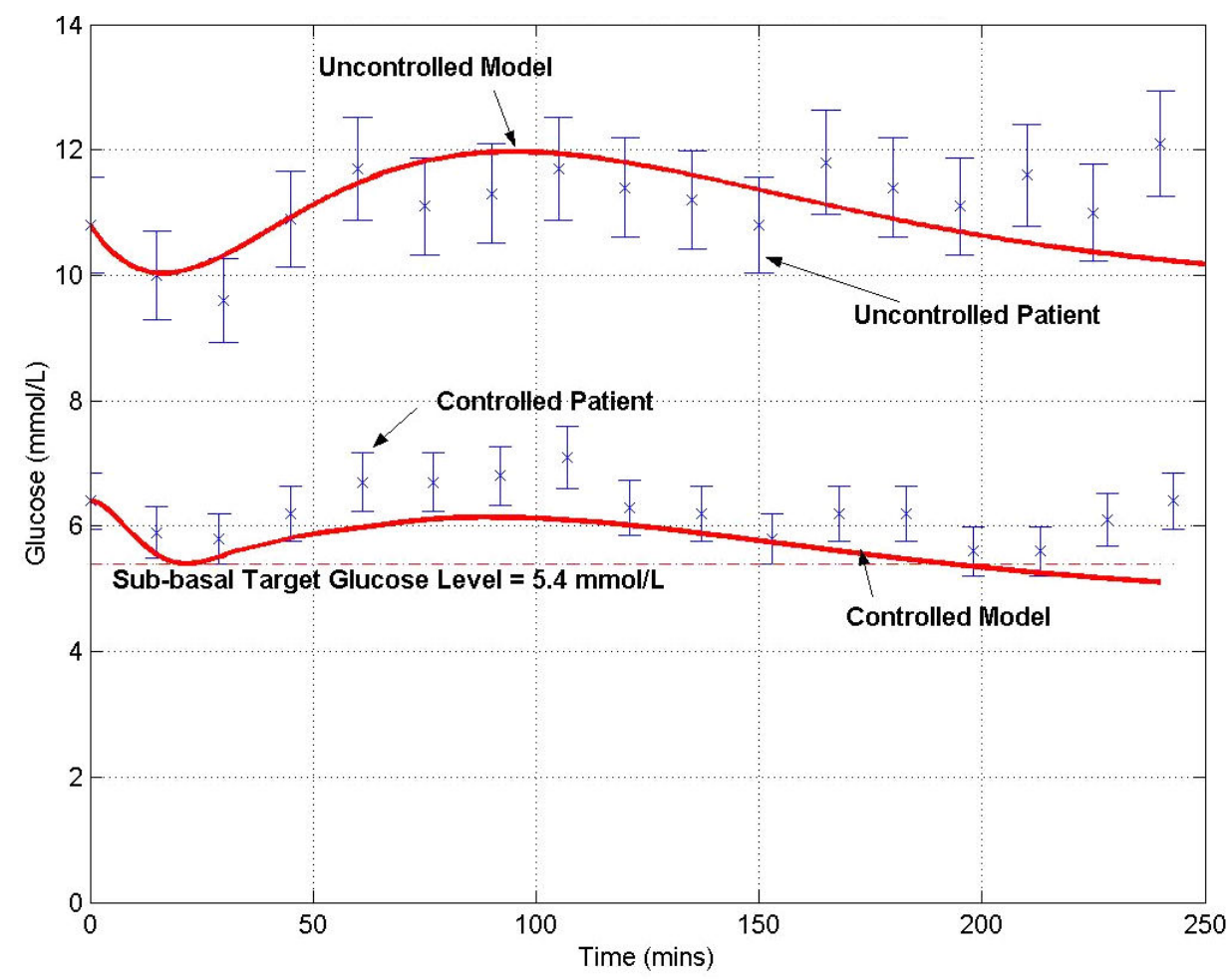

Figure 9: Patient 4 Model vs Measured Glucose - Controlled \& Uncontrolled 75g OGTT 


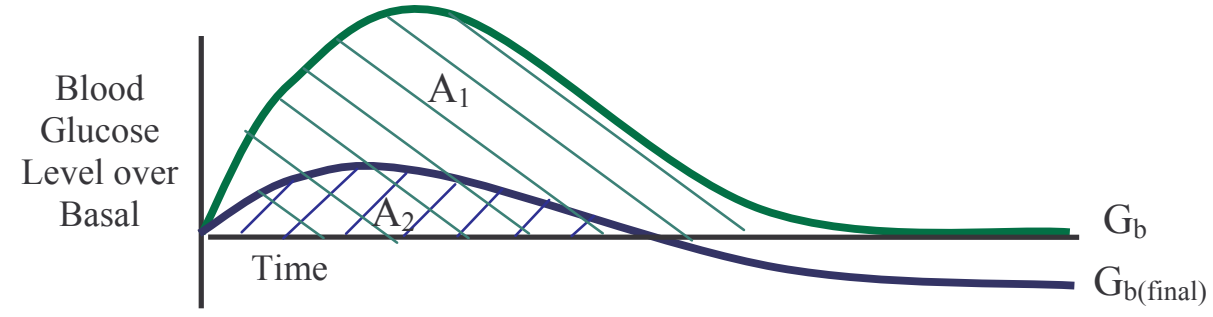

Figure 10: Calculation of Excursion from Basal Glucose Level where $\mathrm{A}_{1}$ represents the area under the Glucose Curve on Day 1 and A2 represents the area under the Glucose Curve on Day 2. 


\begin{tabular}{|c|c|c|c|c|c|c|c|c|}
\hline \multirow{2}{*}{ Patient } & \multirow{2}{*}{ Age } & \multirow{2}{*}{ Condition } & \multirow{2}{*}{$\begin{array}{c}\text { Basal } \\
\text { Glucose } \\
(\mathrm{mmol} / \mathrm{L})\end{array}$} & \multirow{2}{*}{$\begin{array}{c}\text { Peak } \\
\text { Glucose } \\
(\mathrm{mmol} / \mathrm{L})\end{array}$} & \multirow{2}{*}{$\begin{array}{c}\text { Insulin } \\
\text { Level } \\
(p m o l / L)\end{array}$} & \multicolumn{2}{|c|}{$\begin{array}{c}\text { Patient Specific } \\
\text { Parameters }\end{array}$} & \multirow{2}{*}{ Diabetic Type } \\
\hline & & & & & & $\begin{array}{c}p_{1} \\
\left(\min ^{-1}\right)\end{array}$ & $\underset{(\mathrm{L} / \mathrm{mU} / \mathrm{min})}{p_{4}}$ & \\
\hline 1 & 67 & $\begin{array}{l}\text { Kidney } \\
\text { Failure }\end{array}$ & 9.5 & 11.5 & 70 & 0.1549 & 0.0317 & $\begin{array}{l}\text { Hyperglycaemic, } \\
\text { and } \\
\text { hyperinsulinemic }\end{array}$ \\
\hline 2 & 48 & $\begin{array}{c}\text { ARDS, } \\
\text { Tetraplegic }\end{array}$ & 12.5 & 24.5 & 59 & 0.0187 & $1.1 \times 10^{-4}$ & Type 2 \\
\hline 3 & 75 & Head Injury & 13.8 & 22.1 & 3 & 0.0074 & 0.0036 & حype 1 \\
\hline 4 & 59 & Sepsis & 10.8 & 11.8 & 295 & 0.1 & 0.0025 & $\begin{array}{l}\text { Hyperglycaemic, } \\
\text { and } \\
\text { hyperinsulinemic }\end{array}$ \\
\hline
\end{tabular}

Table 1: Patient Summary and Day One Results 


\begin{tabular}{|c|c|c|c|}
\hline Patient & $\begin{array}{c}\text { Model } \\
\text { predicted total } \\
\text { insulin (U) }\end{array}$ & $\begin{array}{c}\text { Day two clinical } \\
\text { trial total } \\
\text { insulin (U) }\end{array}$ & $\begin{array}{c}\text { Percentage difference } \\
\text { between predicted } \\
\text { and infused (\%) }\end{array}$ \\
\hline 1 & 4.50 & 4.43 & -1.55 \\
\hline 2 & 38.24 & 42.65 & 11.53 \\
\hline 3 & 4.90 & 4.50 & -8.16 \\
\hline 4 & 8.90 & 9.80 & 10.11 \\
\hline
\end{tabular}

Table 2: Comparison between predicted insulin and trial insulin infused 


\begin{tabular}{|c|c|c|c|c|c|c|c|}
\hline \multirow[b]{2}{*}{ Patient } & \multicolumn{2}{|c|}{ Day one - OGTT } & \multicolumn{4}{|c|}{ Day two - Clinical Trial } & \multirow[b]{2}{*}{$\mathbf{A}_{2} / \mathbf{A}_{1}$} \\
\hline & $\begin{array}{c}\mathbf{G}_{\mathrm{b}} \\
(\mathrm{mmol} / \mathrm{L})\end{array}$ & $\mathbf{A}_{1}$ & $\begin{array}{c}\mathbf{G}_{\mathrm{b}} \\
(\mathrm{mmol} / \mathrm{L})\end{array}$ & $\mathrm{A}_{2}$ & $\begin{array}{c}\mathbf{G}_{\mathrm{b}(\mathrm{final})} \\
(\mathrm{mmol} / \mathbf{L})\end{array}$ & $\mathbf{G}_{\mathrm{b}(\text { final) }} / \mathbf{G}_{\mathrm{b}}$ & \\
\hline 1 & 9.6 & 292 & 7.2 & 60 & 6.1 & 0.85 & 0.21 \\
\hline 2 & 12.5 & 1524 & 11.6 & 172 & 6.9 & 0.59 & 0.11 \\
\hline 3 & 13.1 & 1082 & 11.1 & 196 & 7.9 & 0.71 & 0.18 \\
\hline 4 & 10.8 & 170 & $\begin{array}{l}6.4 \\
\end{array}$ & 23 & 5.6 & 0.88 & 0.14 \\
\hline
\end{tabular}

Table 3: Comparison of Glucose Excursion for Controlled vs Uncontrolled Data 


\section{List of Figures and Tables}

Figure 1: Blood Glucose Levels

Figure 2: Schematic of the basic dynamics of the human glucose regulatory system.

Figure 3: Response Regimes for PD Blood Glucose Control

Figure 4: Patient 1 Model vs Measured Glucose - Controlled \& Uncontrolled 75g OGTT

Figure 5: Patient 2 Model vs Measured Glucose - Controlled \& Uncontrolled $75 \mathrm{~g}$ OGTT

Figure 6: Patient 2 Insulin Infusion Profile

Figure 7: Patient 2 Glucose Response with Shifted Glucose Input

Figure 8: Patient 3 Model vs Measured Glucose - Controlled \& Uncontrolled 75g OGTT

Figure 9: Patient 4 Model vs Measured Glucose - Controlled \& Uncontrolled 75g OGTT

Figure 10: Calculation of Excursion from Basal Glucose Level

Table 1: Patient Summary and Day One Results

Table 2: Comparison between predicted insulin and trial insulin infused

Table 3: Comparison of Glucose Excursion for Controlled vs Uncontrolled Data 